\title{
Load Capacity of a Gyroid Structure Produced by Selective Laser Melting
}

Pavel Hanzl, Ivana Zetková, Miroslav Zetek

Faculty of Mechanical Engineering, University of West Bohemia. Univerzitní2732/8, 30614 Pilsen. Czech Republic. Email: hanzlp@rti.zcu.cz, mzetek@kto.zcu.cz, zetkova@rti.zcu.cz

The quest for light and stiff structures by industries such as the medical industry and the aerospace industry is the driving force behind developments in additive manufacturing technology. One way to achieve significant weight reduction of components is by deploying periodic porous structures. Various types of porous structure geometries use material with varying efficiency, and therefore vary in their resulting mechanical and physical properties. This article focuses on the lightweight Schoen Gyroid cellular structure, which could offer a suitable combination of the desired mechanical properties. The goal is to determine the effect of the volume fraction on the load capacity of the gyroid structure made of maraging steel using DMLS technology. We conclude from the experimental measurements that the results could be affected by several factors, which are detailed at the end of article.

Keywords: Schoen Gyroid, lattice structures, Rigid Constructions, Additive Manufacturing, Selective Laser Melting

\section{Acknowledgement}

This paper is based upon work sponsored by project SGS-2016-005.

\section{References}

[1] HAO, L., RAYMONT, D., YAN, C., HUSSIEN, A., YONG, P. (2012). Design and Additive Manufacturing of Cellular Lattice Structures, Innovative Developments in Virtual and Physical Prototyping - Bártolo et al. (eds), 2012 Taylor \& Francis Group, London, ISBN 978-0-415-68418-7.

[2] MARCIAN, P., REHOREK, Z., FLORIAN, Z., DLOUHY, I. (2011). Estimation of the Properties Porous Structures by Experiment and Modeling, Chapter 47 in DAAAM International Scientific Book 2011, pp. 573-584, B. Katalinic (Ed.), Published by DAAAM International, ISBN 978-3-901509-84-1, ISSN 1726-9687, Vienna, Austria, DOI: $10.2507 /$ daaam.scibook.2011.47.

[3] TORIMS, T. (2013). The Application of Laser Cladding to Mechanical Component Repair, Renovation and Regeneration, Chapter 32 in DAAAM International Scientific Book 2013, pp. 587-608, B. Katalinic \& Z. Tekic (Eds.), Published by DAAAM International, ISBN 978-3-901509-94-0, ISSN 1726-9687, Vienna, Austria.

[4] KHADERI, S: N., DESHPANDE, V. S., FLECK, N. A. (2014). International Journal of Solids and Structures, Department of Engineering, Cambridge University, Cambridge, International Journal of Solids and Structures 51 (2014) $3866-3877$.

[5] EOS, EOS MarigingSteel MS1, online [2016-06-27], Available from: http://www.innomia.cz/files/tinymce/files/ms1-eng.pdf

[6] YAN, C., HAO, L., HUSSIEN, A., YONG, P., RAYMONT, D. (2014). Advanced lightweight 316L stainless steel cellular lattice structure fabricated via selective laser melting, Published by Elsevier Ltd, Materials and Design 55 (2014) 533-541.

[7] AREMU, A. O. all et. A Comparative finite element study of cubic unit cells for selective laser melting, EPSRC Centre for Innovative Manufacturing in Additive Manufacturing, Faculty of Engineering, University of Nottingham, Nottingham, NG7 2RD, UK.

[8] HANZL, P., ZETKOVÁ, I., MACH, J. (2017). Optimization of the Pressure Porous Sample and its Manufacturability by Selective Laser Melting, Published by Manufacturing Technology, ISSN 1213-2489, Paper number: MT20177.

[9] YAN, C., HAO, L., HUSSEIN, A., YOUNG, P., RAYMONT, D. (2014). Advanced lightweight 316L stainless steel cellular lattice structures fabricated via selective laser melting, Published by Elsevier Ltd, Materials and Design 55 (2014) 533-541

[10] YAN, C., HAO, L., HUSSEIN, A., RAYMONT, D. (2012) Evaluations of cellular lattice structures manufactured using selective laser melting, Published by Elsevier Ltd, International Journal of Machine Tools \& Manufacture 62 (2012) 32-38. 
[11] MURR, L.E. all et. (2010). Characterization of Ti-6Al-4V open cellular foams fabricated by additive manufacturing using electron beam melting, Published by Elsevier Ltd, Materials Science and Engineering A 527 (2010) 1861-1868

[12] YAN, C., HAO, L., HUSSEIN, A., YOUNG, P., HUANG, J. (2015). Microstructure and mechanical properties of aluminium alloy cellular lattice structures manufactured by direct metal laser sintering, Published by Elsevier Ltd, Materials Science \& Engineering A 628 (2015) 238-246.

[13] KUČEROVÁ, L., ZETKOVÁ, I. (2016). Metallography of 3D Printed 1.2709 Tool Steel, Published by Manufacturing Technology, ISSN 1213-2489, Paper number: M201629.

[14] FOUSOVÁ, M., VOJTĚCH, D., KUBÁSEK, J., DVORSKÝ, D., MACHOVÁ, M., 3D Printing as an Alternative to Casting, Forming and Machining Technologies?, Published by Manufacturing Technology, ISSN 1213-2489, Paper number: M2015147.

Copyright (C) 2017. Published by Manufacturing Technology. All rights reserved. 